\title{
The Work of Sergey Alekseevich Lebedev in Kiev and Its Subsequent Influence on Further Scientific Progress There
}

\author{
Zinoviy L. Rabinovich \\ V.M. Glushkov Institute of Cybernetics \\ Kiev, Ukraine
}

\begin{abstract}
The paper represents the memories of an active participant of development of the first Soviet computers in Kiev, Ukraine. The process of creating electronic computing machines such as MECM, BESM and SESM is described in detail. The outstanding role of S.A. Lebedev (head of the research group) is specially stressed as well as his impact on subsequent progress.
\end{abstract}

Keywords: MECM, first Soviet computers, Academician S.A. Lebedev.

\section{Introduction}

In my opinion, the day of the 6 November 1950 was the culmination of Lebedev's entire work in Kiev. This was the day when the MESM-still a prototype-solved its first test problem. That was a miracle! The electronic computing machine was created in the Ukraine!

Here, I will briefly recall what occurred before and after that moment. One should not forget that Sergei Alekseevich Lebedev already was an outstanding scientist [1] before he was appointed to the position as director of the (Kiev) Institute of Electrotechnics. The institute was established with Lebedev's participation. In the beginning, he headed the laboratory of modeling and automatic control there. Then, that laboratory transformed into laboratory of computing machinery at about the end of 1948. That was the time when the development and construction of the small electronic computing machine (MESM) started.

\section{The Birth of MESM}

We could characterize the works performed at the laboratory before that date as the preparation phase for the MESM machine. However, those works themselves also had significant importance, independent of their final objectives. Those were the electronic research and development (with subsequent adjustment) of a special installation for semi-natural modeling and testing of the aircraft flight stabilization systems. That system was made under the order of another research institute. However, the payment received for the work became sound financial backing for initial development of the MESM, which, in fact, began as an experiment launched 
entirely on the laboratory scientists' own initiatives. Nonetheless, the idea to construct this installation was a notable scientific achievement of Sergey Alekseevich. Authoritative scientific sources subsequently highlighted that accomplishment.

Actually, among its other activities, the installation integrated in its structure a special analog computing unit (in fact an analog computer) based on operation amplifiers; it modeled the movements of an aircraft during its flight and provided a dynamic platform that operated with three degrees of freedom. The latter had modeled real angles of movements and distortions. A special high-precession monitoring system, mounted between them, transformed the modeled aircraft flight distortions into power impulses or impacts applied on special torque motors of the platform.

Not only the ideas, but also their technical solutions proposed and realized by Sergey Alekseevich Lebedev were original. That was the reason why the whole project was rightfully named, 'the pioneer one'. I have described it here in such detailed way because few knew about it, as it was overshadowed by his own later brilliant achievements in electronic computer development. However, one should mention that this analog computer, devised by Lebedev for the system, was one of the first Soviet computing machines of the analog type.

Before beginning his practical work on the computer MESM project, Sergei Lebedev personally performed impressive scientific theoretical preparations. He found and developed basic technical solutions for design of the MESM and its principal structural circuits and structure. However, before that, he developed the fundamental general principles for the architectural creation of digital electronic computers - an entirely new kind of computing machinery. In other words, the principles, which are famous as the John von Neumann architectural principles, Lebedev discovered and formulated independently; that is, he invented digital electronic computer all by himself. Construction of the computer lasted two years until the first test run, and one more year until the official governmental commission accepted it and put it into regular operation on 25 December of 1951.

\section{From MESM to BESM}

Rapid performance, considered a necessity to work with absolutely new type of (computing) machinery, became real not only due to Lebedev's own thorough scientific preparations, but almost equally, because of a special project work schedule, or rather a style of the project team's work, introduced by Lebedev and L.N. Dashevskiy during his first scientific deputy at the laboratory. Thus, most of them almost permanently lived in the building of the (secret) research centre, a little off Kiev, where they were assembling the computer. Lebedev himself and his chief engineer P.J. Chernyak lived there with their families. Therefore, those circumstances as well as the unbelievable enthusiasm of all, particularly the young collaborators, became a sure guarantee for their future success.

Another remarkable, and scientifically significant fact regarding the project, was the work of A.A. Lyapunov's team on the new computer. As soon as the MESM was available to perform (although before the official submission), the USSR Academy of Science dispatched an outstanding mathematician and programmer of Moscow Aleksey A. Lyapunov with some other scientists to Lebedev's laboratory for several 
months to conduct mathematical experiments and perform some urgent calculations for defense related problems.

Besides general impulse, given by appearing MESM for the Soviet following computer development progress, as well as its being itself a long-awaited means for solving urgent problems, the MESM played an essential role as a fully operating model (and thus, most important research tool) for development of the following "full-scale" series BESM of "big" computers. Those machines were already in production in Moscow, where Lebedev returned in 1950 to head the Institute of Precision Mechanics and Computer Engineering (IPMCE). Later, this became the most prominent scientific research institute on computers in the USSR. His invaluable experience of the work in Kiev was one of the keys to its further success.

Indeed, the MESM computer did have all the features to receive consideration as a model for the BESM machine. Namely, its architecture already possessed a number of qualitative characteristic features for efficiently developed in later computers. Thus, the ability to perform operations with commands (programming statements) was one of them. This fact enabled Prof. B.N. Malinovskiy of Kiev (then a young assistant of S.A. Lebedev and now famous historian of computing) to define the MESM as the world's first computer with such features.

\section{From BESM to SESM}

Lebedev's phenomenal success in creating the MESM was not the only achievement of his Kiev laboratory. The MESM was a predecessor of the, so-called "big machines" with maximum possible parallelism of information transmission and processing. However, already at that time Sergey Lebedev was also thinking about mini-machines. Consequently, he initiated the development of the sequential arithmetic logic device/unit (ALU) through sequential information transmission and processing. The operational unit was based on dynamic registers on a magnetic drum. That is, it provided maximum economy of electronic components, some of which were not very reliable then. Later, his collaborators discovered that the unit Lebedev devised was intended for a special mathematic mini-computer called SESM, which should solve linear algebraic equations with iteration methods. Later they used this machine for various vector-matrix procedures.

Lebedev's basic idea of the SESM consisted of simultaneous data input and data processing. The sequential ALU of the SESM proved to be quick enough for that purpose. Therefore, considering its destination and considering the principle of combined parallel data input and processing, the SESM could be-and did-defined as a prototype of modern matrix-vector processors, built into the structure of highperformance universal digital electronic computers. Lebedev proposed the general design of the computer, but it was the author (Z.L. Rabinovich), under Lebedev's scientific supervision, who brought it from a detailed project to a functioning machine. Lebedev consulted the Kiev team and he liked the computer they made.

In other words, the SESM, although assembled by Lebedev's collaborators after his departure to Moscow, was part of his achievements of the Kiev period. However, Lebedev himself, being a very modest person, never accepted this idea; since he was working in Moscow then, he did not directly participate in the computer construction. 
The SESM was the second computer made in the Ukraine. It became operational at organizations based on Lebedev's "Laboratory for Modeling". Those were the laboratories of the famous mathematician, Prof. B.V. Gnedenko, which later transformed into the Computer Centre of the AS Ukraine headed by V.M. Glushkov, and still later into the (Kiev) Institute of Cybernetics. The SESM was a civil, scientific oriented computer that operated freely without military secrecy restrictions. Therefore, its appearance caused a positive international resonance. Subsequently, a monograph about the computer published by its designers was translated and distributed in the USA.

Unfortunately, the SESM was the last scientific project in Kiev where Sergey Lebedev was concerned. However, we should not take this literally. After his departure to Moscow, Lebedev did not lose interest in the developments in Kiev. Periodically, he would visit and his former collaborators always asked him personally for an advice or some other assistance. Those relations also manifested themselves through the cooperation between Lebedev's IPMCE of Moscow and Glushkov's Kiev Institute of Cybernetics. These two prominent researchers created world-recognized schools of science that had developed the most advanced electronic computers for mass usage. They distinguished themselves with original progressive technical solutions for problems at the world's highest level. The world scientific community also recognized their achievements as 'Computer Pioneers' by decorating them with subsequent medals, unfortunately awarded posthumously.

The aforementioned cooperation had precisely defined activity fields and it made a very positive influence on the progress of Soviet computing. There was a mutual generation of ideas on increasing computer performance as well as on the development of the "machine intellect" [2] or machine intelligence, on machine realization of the high-level programming languages, on processing knowledge methodology, and developing the dynamical organization of machine computations.

\section{The MIR and ELBRUS Machines}

The second direction of the cooperation proposed by the Kiev Institute of Cybernetics, especially when it concerned realization of high-level language (HLL), was received as a "revolutionary" one. Therefore, it had caused fierce arguing in the beginning. An important supporting role of S.A. Lebedev in its development was remarkable and, to a certain extent, decisive. Moreover, Lebedev developed the principle of structural interpretation of HLL; he preliminarily approved this principle in its general form and tested in details in operation of mini-computer "MIR" designed for engineering calculations and in the experimental model of the patented high performance computer "Ukraine". This principle was also implemented in the multiprocessor computation complex (super-computer) "ELBRUS", considered as an outstanding achievement of the Soviet computer engineering.

Development of the serial computer "MIR" became a similarly outstanding achievement, but in the field of small computers with advanced machine intellect. The mentioned synthesis of ideas of increasing computer performance and of the development of machine intelligence (computer "intellectualizing", so to say) is still in progress. Thus, the Kiev Institute of Cybernetics was developing computers with 
distributed information processing (parallel architecture), realization of a very highlevel programming language, and advanced methods of knowledge processing (socalled an "intellectual solving machine - ISM"). This computer is designed under the supervision of Prof. V.N. Koval, who implemented it with the experience of an earlier developed 'essentially multi-processor complex' with 'multi-conveyor' organization of computation.

AS Ukraine also awarded collaborators of the both institutes (named after Glushkov in Kiev, and after Lebedev in Moscow) with Lebedev's premiums and also for joint projects. This recognition is clear evidence of the friendly relations that existed between the two institutions.

\section{Conclusion}

I would like to finish this narration with an historical phrase of Sergey Alekseevich Lebedev who said on the shore of the lake at Theofania (or Feofania), the Kiev suburb where he made his MESM in the secret laboratory during his visit there. He waved his hand towards the place and said,

"Here, it was here where we started all this".

It would be wonderful if all this could receive further progress, not less intensive then at those times.

\section{End Notes}

1. Soon after the war, Professor Dr. of Technical Sciences, S.A. Lebedev (1902-1974) of Moscow, was elected to the Academy of Sciences Ukraine (AS Ukraine) and he was invited as an experienced specialist to Kiev to participate in the post-war restoration and further development of the science in the republic.

\section{Editors' Note to \#1}

That was a part of the USSR All-Union program of the post-war development.

2. «Machine intellect» - (degree of) technical/structural ability (or perfection) of a computer to realize efficiently algorithms and programmed tasks, including those composed for «artificial intellect».

Editors' Note to \#2

The author, Z.L. Rabinovich (1918-2009), was one of the first, and undoubtedly the leading, Soviet specialists in this field. 